\title{
MEAN CURVATURE 1 SURFACES OF COSTA TYPE IN HYPERBOLIC THREE-SPACE
}

\author{
Celso J. Costa And Vicente F. Sousa Neto
}

(Received February 10, 2000, revised February 5, 2001)

\begin{abstract}
In this paper we prove the existence of families of complete mean curvature one surfaces in the hyperbolic three-space. We show that for each Costa-Hoffman-Meeks embedded minimal surface of positive genus in Euclidean three-space, we can produce, by cousin correspondence, a family of complete mean curvature one surfaces in the hyperbolic three-space. These surfaces have positive genus, three ends and the same group of symmetry of the original minimal surfaces. Furthermore, two of the ends approach the same point in the ideal boundary of hyperbolic three-space and the third end is asymptotic to a horosphere. The method we use to produce these results were developed in a recent paper by W. Rossman, M. Umehara and K. Yamada.
\end{abstract}

1. Introduction. Surfaces of constant mean curvature (CMC) 1 in hyperbolic 3 -space $\boldsymbol{H}^{3}$ share many properties with minimal surfaces in Euclidean 3 -space $\boldsymbol{R}^{3}$, the most important being that both types of surfaces possess a "Weierstrass representation" in terms of holomorphic data [B]. This representation enables us to describe a great number of examples of CMC 1 surfaces in $\boldsymbol{H}^{3}$, which may have branch points. (By $\boldsymbol{H}^{3}$ we mean the unique complete simply-connected 3-manifold with constant sectional curvature -1.)

In [RUY], a method is given for constructing examples without branch points. More precisely, it is proved that for each complete symmetric finite-total-curvature minimal surface in $\boldsymbol{R}^{3}$ with a non-degenerate period problem, there exists a corresponding one-parameter family of complete CMC 1 immersions in $\boldsymbol{H}^{3}$. This provides many examples of CMC 1 surfaces in $\boldsymbol{H}^{3}$, many of which are described in [RUY]. However, as was explained in [RUY, p. 471], the method does not apply directly to show the existence of CMC 1 surfaces corresponding to the original Costa surfaces in $\boldsymbol{R}^{3}$. The goal of this paper is to define good variations of the Weierstrass representation of the minimal Costa-Hoffman-Meeks surfaces and to choose fundamental disks of these surfaces so that the method of [RUY] can be applied, thus showing that corresponding cousin surfaces exist in $\boldsymbol{H}^{3}$. See Theorem 4.1.

By "Costa-Hoffman-Meeks cousin" we mean a CMC 1 surface in $\boldsymbol{H}^{3}$ of positive genus with three ends. The ends asymptotically approach only two points in the ideal boundary at infinity of $\boldsymbol{H}^{3}$. The surfaces in this family are duals (in the sense of [RUY] and [UY3]) of the image under the Lawson correspondence of the original minimal Costa-Hoffman-Meeks surfaces. The Costa-Hoffman-Meeks surfaces in $\boldsymbol{R}^{3}$, which we will call $\boldsymbol{M}_{k}$ [HKM], are complete minimal surfaces with genus $k$ and $k+1$ vertical planes of symmetry, and two

2000 Mathematics Subject Classification. Primary 53A10; Secondary 53A35, 53C42. 
catenoid ends and one flat end. The first example $M_{1}$ was found by Celso Costa [C1] in 1982. Although the $M_{k}$ are embedded, their CMC 1 dual cousins in $\boldsymbol{H}^{3}$ are never embedded, see Figure 4.3. In fact, there is no general relationship between embeddedness of minimal surfaces in $\boldsymbol{R}^{3}$ and their dual cousin surfaces in $\boldsymbol{H}^{3}$. The catenoid is embedded in $\boldsymbol{R}^{3}$, but the catenoid cousin can be either embedded or non-embedded in $\boldsymbol{H}^{3}$; the minimal trinoid cousins in $\boldsymbol{R}^{3}$ are never embedded, but their CMC 1 dual cousins can be either embedded or non-embedded in $\boldsymbol{H}^{3}$. See [RUY] for more on this point.

A part of this work was contained in the doctoral thesis of the second author at Universidade Federal do Ceará (see [N]). He would like to thank Levi Lima and Wayne Rossman for their helpful conversations and guidance.

2. Complete minimal immersions in $\boldsymbol{R}^{3}$. We begin by stating the Weierstrass representation theorem (see $[\mathrm{O}]$ or $[\mathrm{L}])$. This representation is the primary tool for describing complete minimal surfaces in $\boldsymbol{R}^{3}$.

THEOREM 2.1. Let $M$ be a Riemann surface, $\eta$ a holomorphic one-form on $M$, and $g: M \rightarrow C \cup\{\infty\}$ a meromorphic function. Consider the vector valued one-form

$$
\Phi=\left(\phi_{1}, \phi_{2}, \phi_{3}\right):=\left(\left(1-g^{2}\right) \eta, i\left(\left(1+g^{2}\right) \eta, 2 g \eta\right) .\right.
$$

Then

$$
X(p)=\operatorname{Re} \int_{p_{0}}^{p} \Phi
$$

defines a conformal minimal mapping of some covering of $M$ into $\boldsymbol{R}^{3}$. This mapping is welldefined on $M$ if and only if $X$ has no nonzero real period on $M$, that is, if and only if

$$
\operatorname{Period}_{\alpha}(\Phi):=\operatorname{Re} \oint_{\alpha} \Phi=\overrightarrow{0}
$$

for all closed curves $\alpha$ on $M$. This mapping is regular provided the poles of $g$ coincide with the zeros of $\eta$, and, whenever $g$ has a pole of order $l, \eta$ has a zero of order $2 l$. Moreover, $g$ is stereographic projection of the Gauss map $N: M \rightarrow S^{2}$ of $X$. Conversely, every regular conformal minimal immersion $X: M \rightarrow \boldsymbol{R}^{3}$ can be expressed in the form (2.2) for some meromorphic function $g$ and holomorphic one-form $\eta$.

The metric induced on $M$ by $X$ can be expressed as

$$
d s^{2}=\left(1+|g|^{2}\right)^{2} \eta \cdot \bar{\eta},
$$

where - means the symmetric product, and the Gauss curvature $K$ of $X$ is

$$
K=-\frac{4 d g \cdot \bar{d} g}{\left(1+|g|^{2}\right)^{4} \eta \cdot \bar{\eta}} .
$$

Typically, when one wishes to have the Weierstrass representation of $X$ well-defined on $M$, there are parameters in the Weierstrass data $\{M, g, \eta\}$ that one adjusts until (2.3) holds for all closed curves in $M$. This is referred to as the solving the period problem. In the case of finite total curvature $\int_{M} K d A>-\infty$, we have the following theorem: 
THEOREM 2.2 (Osserman-Huber, [L], [O]). Let $X: M \rightarrow \boldsymbol{R}^{3}$ be a complete conformal minimal immersion with finite total curvature. Then the following hold.

(i) $\quad M$ is conformally diffeomorphic to $\bar{M}_{k} \backslash\left\{e_{1}, \ldots, e_{r}\right\}$, where $\bar{M}_{k}$ is a closed Riemann surface of genus $k$ and $e_{1}, \ldots, e_{r}$ are points in $\bar{M}_{k}, r \geq 1$.

(ii) The Gauss map $N: M \rightarrow S^{2}$, which is meromorphic on $M$, extends to a meromorphic function on $\bar{M}_{k}$. The one-form $\eta$, which is holomorphic on $M$, extends to a meromorphic one-form on $\bar{M}_{k}$.

(iii) The total curvature is an integer multiple of $4 \pi$ and satisfies

$$
\int_{M} K d A \leq-4 \pi(k+r-1),
$$

where $k$ and $r$ are the integers defined in statement (i).

If $D_{j} \subset \bar{M}_{k}$ is a punctured neighborhood of $e_{j}$, then the image $X\left(D_{j}\right)$ of $D_{j}$ is an end of $M$, which we denote by $E_{j} . E_{j}$ is an embedded end if and only if $\eta$ has a pole of order two at $e_{j}[\mathrm{JM}]$. Since $g$ is the stereographic projection of the Gauss map $N$ of $X$, we can suppose, after a rotation of $X$ in $\boldsymbol{R}^{3}$ if necessary, that $g\left(e_{j}\right)=0$. If the end $E_{j}$ is embedded, then, near $e_{j}$, we have the local expression

$$
g(z)=a_{n} z^{n}+o\left(z^{n+1}\right), \quad a_{n} \neq 0, n \geq 1 \quad \text { and } \quad \eta(z)=\frac{b}{z^{2}}+o(1), b \neq 0 .
$$

We say that an embedded end $E_{j}$ is a catenoid end if $n=1$ and is a flat end of order $n-1$ if $n>1$. It holds that:

1. All of the ends of $M$ are embedded if and only if the equality holds in (iii) of Theorem 2.2.

2. If no two ends of $M$ intersect, then, after a rotation of $\boldsymbol{R}^{3}$, the Gauss map $N$ satisfies $N\left(e_{j}\right)=(0,0, \pm 1)$ for all $j=1, \ldots, r$.

It is clear that if $X: M \rightarrow \boldsymbol{R}^{3}$ is an embedding, then the above two conditions must hold. However, these conditions are not sufficient to imply that $X$ is an embedding. One can see examples and a discussion on this in [W1], [W2], [HK]. However, when $M$ is embedded and complete and has finite total curvature, it follows that outside of a sufficiently large compact set of $\boldsymbol{R}^{3}, M$ is asymptotic to a finite number of half-catenoids and planes, which may be assumed to have vertical normals.

The Schwarz reflection principle for minimal surfaces is also central to the arguments that we will be making. Before stating it, we recall some relevant facts. A curve on any surface in $\boldsymbol{R}^{3}$ is a straight line if and only if both the geodesic curvature and the normal curvature vanish. A principal curve on a surface that is not a straight line has the property that it is a geodesic if and only if it lies in a plane orthogonal to the surface. For the surface $X(M)$, expressing the shape operator $S=D X \cdot D N$ in terms of $g$ and $\eta:=f d z$ for some holomorphic function $f$ and holomorphic coordinate $z$, we conclude that a curve $c$ on $X(M)$ is

asymptotic $\Longleftrightarrow g^{\prime} f\left(d z\left(c^{\prime}(t)\right)\right)^{2} \in i \boldsymbol{R}, \quad$ and $\quad$ principal $\Longleftrightarrow g^{\prime} f\left(d z\left(c^{\prime}(t)\right)\right)^{2} \in \boldsymbol{R}$. 
THEOREM 2.3 (Schwarz reflection principle). If a minimal surface is bounded by a line segment $\ell$, it may be extended by rotation by angle $\pi$ about $\ell$ to a smooth minimal surface containing $\ell$ in its interior. If a minimal surface with boundary contains a planar geodesic $c$ in its boundary, then this surface can be extended smoothly across $c$ by reflecting through the plane containing $c$.

3. Costa surfaces in $\boldsymbol{R}^{3}$. In this section we briefly review the construction and some properties of the surfaces $M_{k}, k \in N$. Each of the surfaces $M_{k}$ is conformally equivalent to a Riemann surface of genus $k$ punctured at three points. The surfaces $M_{k}$ have two catenoid ends and one flat end. The example with $k=1$ was found by Costa, who constructed it using the classical Weierstrass $\wp$-function. He also proved that this surface satisfies all known necessary conditions for embeddedness. Hoffman and Meeks were able to prove that $M_{k}$ is embedded and highly symmetric (see [HM1] for $k=1$ and [HM2] for general $k$ ).

We will use the Weierstrass Representation Theorem 2.1, for which we need a Riemann surface, a meromorphic map, and a holomorphic one-form. We start by describing the complex structure of $M_{k}$. Consider, for each $k \in N$, the compact Riemann surface $\bar{M}_{k}$ associated to the irreducible polynomial

$$
P(z, w)=w^{k+1}-z^{k}(z+1)(z-1) .
$$

Let $p_{0}=(0,0), p_{-1}=(-1,0), p_{+1}=(1,0)$, and $p_{\infty}=(\infty, \infty)$ be the singular points of $P(z, w)$. Then

$$
\bar{M}_{k}=\left\{(z, w) \in(\boldsymbol{C} \cup\{\infty\})^{2} \mid P(z, w)=0\right\}
$$

is the compact Riemann surface associated to $P(z, w)$. One can see that $\bar{M}_{k}$ is a $(k+1)$ sheeted ramified covering of the Riemann sphere $C \cup\{\infty\}$, with genus $k$. Now, consider

$$
M_{k}=\bar{M}_{k}-\left\{p_{-1}, p_{+1}, p_{\infty}\right\},
$$

the Riemann surface obtained by puncturing $\bar{M}_{k}$ in three points. (Note that, for simplicity of notation, we are now referring to both the Riemann surface in the Weierstrass data and the actual minimal surface in $\boldsymbol{R}^{3}$ by the same notation $M_{k}$.) The next theorem defines the conformal minimal immersion of $M_{k}$ into $\boldsymbol{R}^{3}$.

THEOREM 3.1 (Hoffman and Meeks [HM]). Let $M_{k}$ and $\bar{M}_{k}$ be the Riemann surfaces given by (3.4) and (3.5), and let

$$
\eta=\left(\frac{z}{w}\right)^{k} d z=\frac{w}{(z+1)(z-1)} d z, \quad g=\frac{a}{w}, \quad a \in \boldsymbol{R}^{+},
$$

be a meromorphic one-form and a meromorphic function on $\bar{M}_{k}$. Consider the vector-valued one-form $\Phi$ as in (2.1). Then there exists a unique $a>0$ such that $X(p)=\operatorname{Re} \int_{p_{0}}^{p} \Phi: M_{k} \rightarrow$ $\boldsymbol{R}^{3}$ is a complete properly-embedded conformal minimal immersion with finite total curvature of $M_{k}$ into $\boldsymbol{R}^{3}$.

After suitable rotations and translations of $\boldsymbol{R}^{3}$, the surface $M_{k}$ has the following properties: 
1) The total curvature of $M_{k}$ is $-4 \pi(k+2)$.

2) $M_{k}$ has one flat end between its top and bottom catenoids ends. The flat end is asymptotic to the $\left(x_{1}, x_{2}\right)$-plane.

3) $\quad M_{k}$ intersects the $\left(x_{1}, x_{2}\right)$-plane in $k+1$ straight lines, which meets at equal angles at the origin.

4) The symmetry group of $M_{k}$ is the dihedral group with 4(k+1) elements generated by reflection in $k+1$ vertical planes of symmetry meeting in the $x_{3}$-axis, and rotation about one of the lines in (3).

5) $M_{k}$ may be decomposed into $4(k+1)$ congruent pieces, each of which is a graph.

6) $M_{k}$ is the unique properly-embedded minimal surface of genus $k$ with three ends, finite total curvature, and a symmetry group containing $4(k+1)$ or more elements.

The symmetry of the surface is used to show that there is only one period condition. The value of the constant $a$ in Theorem 3.1 for which the period problem is solved is

$$
a=\sqrt{\frac{2 \int_{0}^{1} t^{k /(k+1)}\left(1-t^{2}\right)^{-k /(k+1)} d t}{\int_{0}^{1}\left(t^{k}\left(1-t^{2}\right)\right)^{-1 /(k+1)} d t} .}
$$

4. Costa type surfaces in $\boldsymbol{H}^{3}$. In this section we will study the period problem for the surfaces $M_{k}$. By using the symmetry of the complex structure of $M_{k}$, and extending the fundamental domains of these surfaces, we will show directly that all surfaces $M_{k}$ have nondegenerate period problems in the sense of [RUY]. So, we achieve that for each $M_{k}$ there exist a one-parameter family of cousin surfaces in $\boldsymbol{H}^{3}$. We will need the following definition.

Definition 4.1. A complete minimal immersion $f: \mathcal{S} \rightarrow \boldsymbol{R}^{3}$ is symmetric if there is a subregion $D \subset f(\mathcal{S})$ that is a disk bounded by non-straight planar geodesics and the interior of $D$ does not contain any non-straight planar geodesics.

Note that if $f$ is symmetric with subdisk $D$, then by the Schwartz reflection principle, $D$ generates the entire surface by reflections across planes containing boundary planar geodesics.

4.1. The surfaces $M_{k}$. An important property of the surfaces $X: M_{k} \rightarrow R^{3}$ is that a fundamental pieces of the surfaces $M_{k}$ are bounded by two planar geodesics and a single straight ray. A such fundamental pieces generates the entire surface by the action of the dihedral group. Consider the disk $D_{k}$ that is the union of this fundamental piece and its rotation by angle $\pi$ about the boundary ray. With respect to this disk $D_{k}$, the immersion $X$ of Theorem 3.1 is symmetric, see Definition 4.1 and Figure 4.1.

In order to apply the results of [RUY], $D_{k}$ must be bounded only by nonstraight planar geodesics. Hence we must use the union of two fundamental pieces with a common boundary ray, and to add extra reals parameters $\mu$ and $\delta$ to the Weierstrass data of $X$.

With the introduction of these parameters, the period problem in the sense of [RUY] becomes two-dimensional. As before, let $\bar{M}_{k}$ be the compact Riemann surface given by 
$\bar{M}_{k}=\left\{(z, w) \in(\boldsymbol{C} \cup\{\infty\})^{2} \mid w^{k+1}=z^{k}\left(z^{2}-1\right)\right\}$ and

$$
M_{k}=\bar{M}_{k} \backslash\left\{p_{+1}, p_{-1}, p_{\infty}\right\},
$$

where $p_{ \pm 1}=( \pm 1,0), p_{\infty}=(\infty, \infty)$, and now let the Weierstrass data be given by

$$
\eta=\frac{w}{\left(z^{2}-1\right)} d z, \quad g_{\lambda}=\frac{\mu+\delta z}{w}
$$

where $\lambda=(\mu, \delta),|\mu-a|<\varepsilon,|\delta|<\varepsilon$, for some fixed $\varepsilon>0$. Here $a>0$ is as given in (3.7).

Let $\tilde{M}_{k}$ be the universal cover of $M_{k}$. We have the following lemma:

Lemma 4.1. Consider $X_{\lambda}(p)=\operatorname{Re} \int_{p_{0}=(0,0)}^{p} \Phi_{\lambda}: \tilde{M}_{k} \rightarrow \boldsymbol{R}^{3}$, where $\Phi_{\lambda}, g_{\lambda}$ and $\eta$ are defined by the equation (2.1). Let $S_{k}$ be a sheet of $\tilde{M}_{k}$ over $\{z \in C \mid \operatorname{Im} z \geq 0\} \backslash\{1,-1\}$, where $\left(1 / 2, e^{i \pi /(k+1)} \tilde{w}(1 / 2)\right) \in S_{k}$ is such that $\tilde{w}(1 / 2)>0$. Then, restricting the paths of integration to lie in this sheet, we have the curves

i) $S_{1,1}(\lambda):=X_{\lambda}([0,1)) \subset P_{1}$

$$
:=\left\{\left(x_{1}, x_{2}, x_{3}\right) \in \boldsymbol{R}^{3} \mid x_{2} \cos \frac{\pi}{(k+1)}+x_{1} \sin \frac{\pi}{(k+1)}=0\right\},
$$

ii) $S_{1,2}(\lambda):=X_{\lambda}((-\infty,-1)) \subset \tilde{P}_{1}(\lambda)$

$$
:=\left\{\left(x_{1}, x_{2}, x_{3}\right) \in \boldsymbol{R}^{3} \mid x_{2} \cos \frac{\pi}{(k+1)}+x_{1} \sin \frac{\pi}{(k+1)}=c_{1}(\lambda)\right\},
$$

iii) $S_{2,1}(\lambda):=X_{\lambda}((-1,0]) \subset P_{2}:=\left\{\left(x_{1}, x_{2}, x_{3}\right) \in \boldsymbol{R}^{3} \mid x_{2}=0\right\}$,

iv) $S_{2,2}(\lambda):=X_{\lambda}((1, \infty)) \subset \tilde{P}_{2}(\lambda):=\left\{\left(x_{1}, x_{2}, x_{3}\right) \in \boldsymbol{R}^{3} \mid x_{2}=c_{2}(\lambda)\right\}$.

The constants $c_{1}$ and $c_{2}$ depend only $\lambda$. Moreover, the curves $S_{i, j}(\lambda)$ are planar geodesics.

Proof. We identify $\boldsymbol{R}^{3} \simeq \boldsymbol{C} \times \boldsymbol{R}$. So, $X_{\lambda}=\left(X_{1}+i X_{2}, X_{3}\right)_{\lambda}$ and

$$
\left(X_{1}+i X_{2}\right)_{\lambda}(p)=\overline{\int_{p_{0}}^{p} \eta}-\int_{p_{0}}^{p} g_{\lambda}{ }^{2} \eta .
$$

So, if $\beta_{1}(t)=\left(t, e^{i \pi /(k+1)} \tilde{w}(t)\right) \in S_{k}$, where $\tilde{w}(t)>0$, and $0 \leq t<1$, we obtain

$$
\begin{aligned}
\gamma_{1}(t) & =\left(X_{1}+i X_{2}\right)_{\lambda}\left(\beta_{1}(t)\right)=\overline{\int_{0}^{t} \frac{e^{i \pi /(k+1)} \tilde{w}(t) d t}{t^{2}-1}}-\int_{0}^{t} \frac{(\mu+\delta t)^{2} d t}{\left(t^{2}-1\right) e^{i \pi /(k+1)} \tilde{w}(t)} \\
& =e^{-i \pi /(k+1)} \int_{0}^{t}\left(\tilde{w}(t)-\frac{(\mu+\delta t)^{2}}{\tilde{w}(t)}\right) \frac{d t}{\left(t^{2}-1\right)} .
\end{aligned}
$$

This last expression shows that $\gamma_{1}(t)$ lies in $P_{1} \cap\left\{x_{3}=0\right\}$ and proves (i). 
On the other hand, let $\beta_{2}(t)=\left(t,-e^{i \pi /(k+1)} \tilde{w}(t)\right) \in S_{k}$, where $-\infty<t<-1$ and $\tilde{w}(t)>0$. Then if $p^{*}=\left(-2,-e^{i \pi /(k+1)} \tilde{w}(t)\right) \in S_{k}, \tilde{w}(t)>0$, we obtain

$$
\begin{aligned}
\gamma_{2}(t) & =\left(X_{+} i X_{2}\right)_{\lambda}\left(\beta_{2}(t)\right) \\
& =\left(X_{1}+i X_{2}\right)_{\lambda}\left(p^{*}\right)-\overline{\int_{-2}^{t} \frac{e^{i \pi /(k+1)} \tilde{w}(t)}{t^{2}-1}}+\int_{-2}^{t} \frac{(\mu+\delta t)^{2} d t}{\left(t^{2}-1\right) e^{i \pi /(k+1)} \tilde{w}(t)} \\
& =c_{1}-e^{-i \pi /(k+1)} \int_{-2}^{t}\left(\tilde{w}(t)-\frac{(\mu+\delta t)^{2}}{\tilde{w}(t)}\right) \frac{d t}{\left(t^{2}-1\right)} .
\end{aligned}
$$

So, $\gamma_{2}^{\prime}(t) \in P_{1} \cap\left\{x_{3}=0\right\}$ for every $-\infty<t<-1$ and this proves (ii).

The proof of (iii) and (iv) is very similar to that of (i) and (ii). It is sufficient to observe that $(-1 / 2,-\tilde{w}(-1 / 2))$ and $(2, \tilde{w}(2))$ are the points of $S_{k}$ over $z=-1 / 2$ and $z=2$, respectively, where $\tilde{w}(-1 / 2)>0$ and $\tilde{w}(2)>0$.

Finally, we observe that if $c(t)=(t, w(t)) \in S_{k}$ with $t \neq \pm 1$, then, by using $\eta=$ $f(z) d z, g_{\lambda}=g_{\lambda}(z)$ as a local representation of $\eta$ and $g_{\lambda},(z, w) \in S_{k}$, we arrive at

$$
g_{\lambda}^{\prime} f\left(d z\left(c^{\prime}(t)\right)\right)^{2}=\left\{\frac{\delta}{t^{2}-1}-\frac{(\mu+\delta t)\left[(k+2) t^{2}-k\right]}{(k+1) t\left(t^{2}-1\right)^{2}}\right\}\left(d z\left(c^{\prime}(t)\right)\right)^{2} \in \boldsymbol{R} .
$$

This implies that the $S_{i j}(\lambda)$ are principal curves, and hence they are planar geodesics.

By Lemma 4.1, the boundary of the disk $D_{k}(\lambda)=X_{\lambda}\left(S_{k}\right)$ is composed of four planar geodesics. Moreover, we know from [HM2] that if $\lambda_{0}=(a, 0)$, with $a$ as defined in (3.7), then the period problem in the sense of the formula (2.3) is solved. In this situation, the plane $P_{1}$ coincides with the plane $\tilde{P}_{1}\left(\lambda_{0}\right)$, and $P_{2}$ coincides with $\tilde{P}_{2}\left(\lambda_{0}\right)$ (see Figure 4.1 ), and by the Schwarz reflection principle, $D_{k}\left(\lambda_{0}\right)$ generates the entire minimal surface $M_{k}$.

Now, let

$$
\begin{aligned}
& \mathcal{D}_{1}(\lambda):=\left\{\text { oriented distance between the planes } P_{1} \text { and } \tilde{P}_{1}(\lambda)\right\} . \\
& \mathcal{D}_{2}(\lambda):=\left\{\text { oriented distance between the planes } P_{2} \text { and } \tilde{P}_{2}(\lambda)\right\} .
\end{aligned}
$$

Defining $\mathcal{D}: \boldsymbol{R}^{2} \rightarrow \boldsymbol{R}^{2}$ by $\mathcal{D}(\lambda)=\left(\mathcal{D}_{1}(\lambda), \mathcal{D}_{2}(\lambda)\right)$, we can then say that the period problem with respect to the disc $D_{k}\left(\lambda_{0}\right)$ is non-degenerate if $\mathcal{D}\left(\lambda_{0}\right)=0$ and the Jacobian of $\mathcal{D}$ at $\lambda_{0}$ is not null. (This definition is sufficient for our considerations, and is a special case of a more general definition in [RUY]).

In order to prove that the period problem for the $D\left(\lambda_{0}\right)$ is non-degenerate, we will consider $\alpha_{1}(t), \alpha_{2}(t) \subseteq \tilde{M}_{k}$ curves in the sheet $S_{k}$ chosen in Lemma 4.1 such that their projections to the $z$-coordinate are $-1+e^{i t}$ and $1+e^{i t}, 0 \leq t \leq \pi$, respectively. Observe that $N_{1}=(\sin (\pi /(k+1)), \cos (\pi /(k+1)), 0)$ is a normal vector of $P_{1}$. Then, with the 


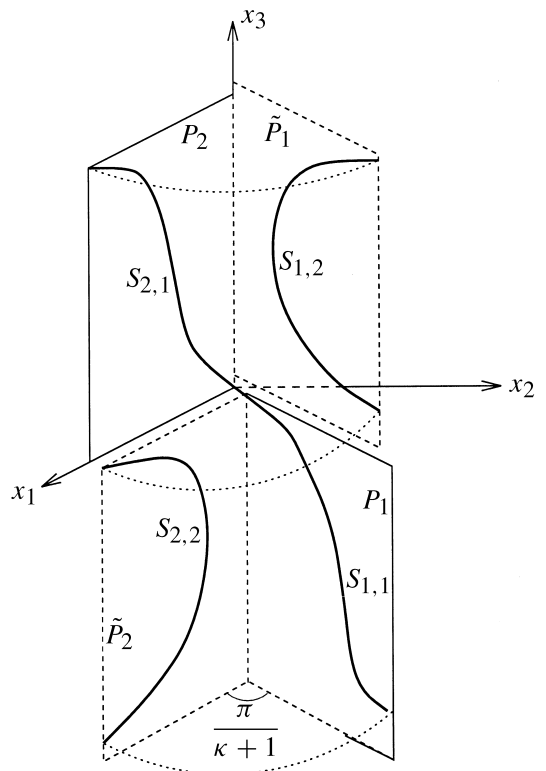

FIGURE 4.1. The pertubed disk $D_{k}(\lambda)$.

identification $\boldsymbol{R}^{3} \cong \boldsymbol{C} \times \boldsymbol{R}, N_{1}=i e^{-i \pi /(k+1)}$, and we conclude that

$$
\mathcal{D}_{1}(\lambda)=-\operatorname{Re}\left[\left(X_{1}+i X_{2}\right)_{\lambda}\left(\alpha_{1}(\pi)\right) i e^{i \pi /(k+1)}\right]
$$

$$
=\operatorname{Re}\left[\overline{\int_{\alpha_{1}} i e^{-i \pi /(k+1)} \eta}+\int_{\alpha_{1}} i e^{i \pi /(k+1)} g_{\lambda}{ }^{2} \eta\right] .
$$

In the same way we find that

$$
\mathcal{D}_{2}(\lambda)=\operatorname{Re} \int_{\alpha_{2}} i\left(1+g_{\lambda}{ }^{2}\right) \eta .
$$

On the other hand, it is easy to compute that:

$$
\begin{aligned}
& \frac{\partial \mathcal{D}_{1}}{\partial \mu}(a, 0)=2 a \operatorname{Re} \int_{\alpha_{1}} \frac{i e^{i \pi /(k+1)} d z}{\left(z^{2}-1\right) w}, \quad \frac{\partial \mathcal{D}_{2}}{\partial \mu}(a, 0)=2 a \operatorname{Re} \int_{\alpha_{2}} \frac{i d z}{\left(z^{2}-1\right) w}, \\
& \frac{\partial \mathcal{D}_{1}}{\partial \delta}(a, 0)=2 a \operatorname{Re} \int_{\alpha_{1}} \frac{i e^{i \pi /(k+1)} z d z}{\left(z^{2}-1\right) w}, \quad \frac{\partial \mathcal{D}_{2}}{\partial \delta}(a, 0)=2 a \operatorname{Re} \int_{\alpha_{2}} \frac{i z d z}{\left(z^{2}-1\right) w} .
\end{aligned}
$$

With these expressions for the partial derivative of the map $\mathcal{D}$, we will prove the following Lemma.

LEMmA 4.2. The Jacobian of the map $\mathcal{D}=\left(\mathcal{D}_{1}, \mathcal{D}_{2}\right)$ is non-null at the point $\lambda_{0}$. 
Proof. We observe that $\zeta: M_{k} \longrightarrow M_{k}$ defined by $\zeta(z, w)=\left(-\bar{z},-e^{i \pi /(k+1)} \bar{w}\right)$ is an anticonformal diffeomorphism such that $\zeta\left(S_{k}\right)=S_{k}$, where $S_{k}$ is the fixed sheet of $\tilde{M}_{k}$ over $\{z \in \boldsymbol{C} \mid \operatorname{Im} z \geq 0\} \backslash\{-1,1\}$. To see this, observe that $(i, w(i))=\left(i, e^{i \pi(k+2) / 2(k+1)} \tilde{w}(i)\right) \in$ $S_{k}, \tilde{w}(i)>0$, is a fixed point for $\zeta$. Furthermore $\zeta\left(\alpha_{1}\right)=-\alpha_{2}$. Then,

$$
\frac{\partial \mathcal{D}_{1}}{\partial \mu}(a, 0)=2 a \operatorname{Re} \int_{\zeta\left(-\alpha_{2}\right)} \frac{i e^{i \pi /(k+1)} d z}{\left(z^{2}-1\right) w}=2 a \operatorname{Re} \int_{\alpha_{2}} \frac{-i d \bar{z}}{\left(\bar{z}^{2}-1\right) \bar{w}}=\frac{\partial \mathcal{D}_{2}}{\partial \mu}(a, 0) .
$$

Also, in the same way, we find that

$$
\frac{\partial \mathcal{D}_{1}}{\partial \delta}(a, 0)=-2 a \operatorname{Re} \int_{\alpha_{2}} \frac{i z d z}{\left(z^{2}-1\right) w}=-\frac{\partial \mathcal{D}_{2}}{\partial \delta}(a, 0) .
$$

Then, to conclude the proof of Lemma, it suffices to prove the following assertion:

Assertion: For every $k \in \boldsymbol{Z}, k>0, I_{1}>0$ and $I_{2}<0$, where

$$
I_{j}=2 \operatorname{Re} \int_{\alpha_{2}} \frac{i z^{j-1} d z}{\left(z^{2}-1\right) w}, \quad j=1,2 .
$$

In order to prove this assertion, we define for each $l, p \in \boldsymbol{Z}$ the meromorphic functions

$$
\varphi_{l, p}=\frac{z^{l+1} w^{p+1}}{z^{2}-1} .
$$

Then

$$
d \varphi_{l, p}=(p+1) \frac{z^{l+1}}{z^{2}-1} w^{p} d w+\frac{(l+1) z^{l}\left(z^{2}-1\right)-2 z^{l+2}}{\left(z^{2}-1\right)^{2}} w^{p+1} d z .
$$

As

$$
(k+1) w^{k} d w=\left(k z^{k-1}\left(z^{2}-1\right)+2 z^{k+1}\right) d z,
$$

we find

$$
d \varphi_{l, p}=\frac{p+1}{k+1}\left(k z^{k+l}+\frac{2 z^{k+l+2}}{z^{2}-1}\right) w^{p-k} d z+\left(\frac{(l+1) z^{l}}{z^{2}-1}-\frac{2 z^{l+2}}{\left(z^{2}-1\right)^{2}}\right) w^{p+1} d z .
$$

The last expression implies that

$$
(k+1) d \varphi_{-k, k-1}+\frac{2 d z}{\left(z^{2}-1\right) w}=-\frac{d z}{w},
$$

and

$$
(k+1) d \varphi_{-k+1, k-1}+\frac{2 z d z}{\left(z^{2}-1\right) w}=\frac{k z d z}{w} .
$$

Observe that,

$$
\varphi_{-k, k-1}\left(\alpha_{2}(0)\right)=\varphi_{-k, k-1}\left(\alpha_{2}(\pi)\right)=\varphi_{-k+1, k-1}\left(\alpha_{2}(0)\right)=\varphi_{-k+1, k-1}\left(\alpha_{2}(\pi)\right)=0 .
$$

Then, by (4.16) and (4.17), we get that

$$
I_{1}=-\operatorname{Re} \int_{\alpha_{2}} \frac{i}{w} d z, \quad I_{2}=k \operatorname{Re} \int_{\alpha_{2}} \frac{i z}{w} d z .
$$


Since $d z / w$ and $z d z / w$ are analytic differentials on $M_{k}$, we can collapse the path $\alpha_{2}$ over the interval $[0,2]$ (see Figure 4.2), to find that

$$
\begin{aligned}
I_{1} & =\operatorname{Re} \int_{[0,2]} \frac{i}{w} d z=\operatorname{Re} \int_{0}^{1} \frac{i e^{-i \pi /(k+1)} d t}{\left(t^{k}\left(1-t^{2}\right)\right)^{1 /(k+1)}} \\
& =\sin (\pi /(k+1)) \operatorname{Re} \int_{0}^{1} \frac{d t}{\left(t^{k}\left(1-t^{2}\right)\right)^{1 /(k+1)}}>0,
\end{aligned}
$$

and

$$
\begin{aligned}
I_{2} & =\operatorname{Re} \int_{[0,2]} \frac{-k i t}{w} d t=\operatorname{Re} \int_{0}^{1} \frac{-k i t e^{-i \pi /(k+1)} d t}{\left(t^{k}\left(1-t^{2}\right)\right)^{1 /(k+1)}} \\
& =\sin (\pi /(k+1)) \operatorname{Re} \int_{0}^{1} \frac{-k t d t}{\left(t^{k}\left(1-t^{2}\right)\right)^{1 /(k+1)}}<0,
\end{aligned}
$$

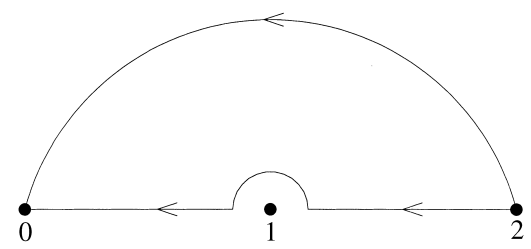

FIGURE 4.2.
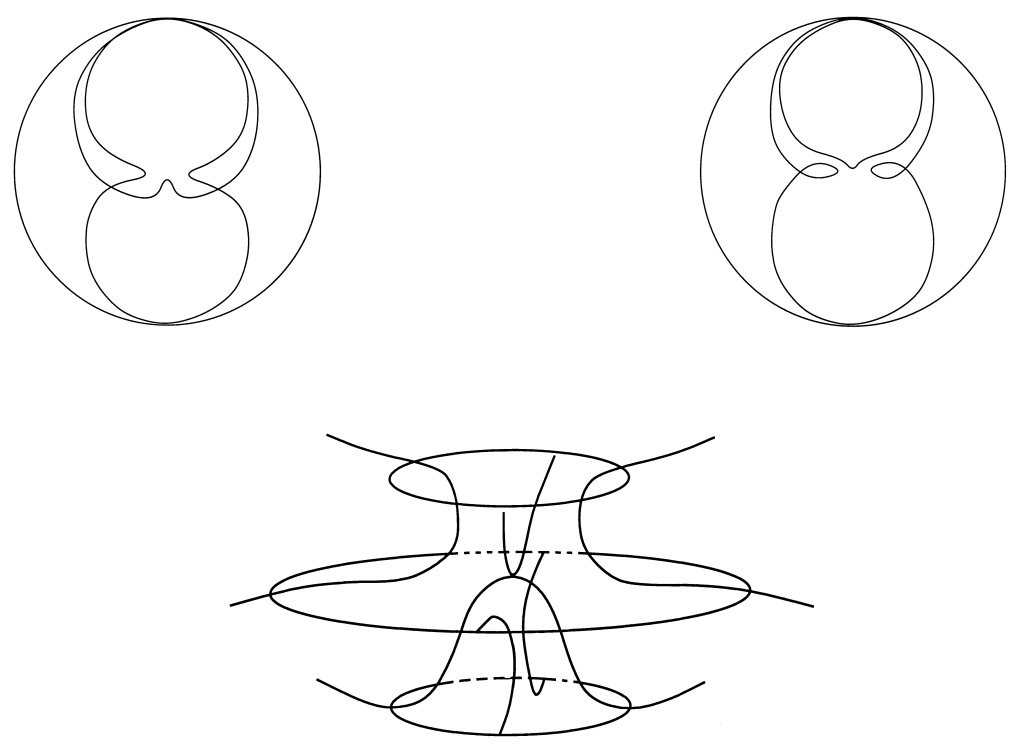

FIgURE 4.3. The surface corresponding to $X\left(M_{1}\right)$ in $\boldsymbol{H}^{3}$ with the Poincaré model, and slices in the $x_{1} x_{3}$-plane and $x_{2} x_{3}$-plane. 
This concludes the proof of the Assertion and completes the proof of the Lemma.

Now we can prove the following Theorem:

THEOREM 4.1. For each $k \in \boldsymbol{Z}, k \geq 1$, there exists a corresponding one-parameter family of CMC 1 Costa type surfaces in $\boldsymbol{H}^{3}$. Each member of the family is complete, with three regular embedded ends, and of finite total curvature and genus $k$. Two of the ends approach the same point in the ideal boundary of $\boldsymbol{H}^{3}$, and are asymptotic to catenoid cousin ends. The third end is asymptotic to a horosphere.

Proof. The first two affirmations of this Theorem are an immediate consequence of Lemmas 4.1 and 4.2, and Theorem 5.10 in [RUY]. To prove the last two sentences, we observe that the Hopf differential $Q:=d g \eta$ of the Costa-Hoffman-Meeks cousin is the same as for the original minimal surfaces $M_{k}$. Furthermore, $Q$ has a pole of order -2 at the two catenoid cousin ends, and a pole of order $k-1$ at the third end. Then one can apply the following result that appears in the appendix of [LR]: an embedded end with finite total curvature is asymptotic to a catenoid cousin end if and only if the order of $Q$ is -2 , and is asymptotic to a horosphere if and only if the order of $Q$ is greater than or equal to -1 . This completes the proof of the Theorem.

\section{REFERENCES}

[B] R. BRYANT, Surfaces of mean curvature one in hyperbolic space, Astérisque No. 154-155 (1987), 321-347.

[C1] C. CostA, Imersões mínimas completas em $\boldsymbol{R}^{3}$ de gênero um e curvatura total finita, Ph. D. Thesis, IMPA, Rio de Janeiro, Brasil, 1982.

[C2] C. Costa, Example of a complete immersion in $\boldsymbol{R}^{3}$ of genus one and three embedded ends Bol. Soc. Brasil. Mat. 15 (1984), 47-54.

[JM] L. P. JORGE AND W. MEEKs, The topology of complete minimal surfaces of finite total Gaussian curvature, Topology 22 (1983), 203-221.

[LR] L. L. Lima AND W. Rossman, On the index of constant mean curvature 1 surfaces in hyperbolis space, Indiana Univ. Math. J. 47 (1998), 685-723.

[N] V. F. S. Neto, Costa type surfaces in hyperbolic space, An. Acad. Brasil. Ciênc. 71 (1999), 333-338.

[O] R. Osserman, A Survey of Minimal Surfaces, Dover Publications, New York, 2nd edition, 1986.

[RUY] W. Rossman, M. UMEHARA AND K. YAmADA, Irreducible constant mean curvature 1 surfaces in hyperbolic space with positive genus, Tôhoku Math. J. 49 (1997), 449-484

[ST] R. SA EARP AND E. TOUBIANA, On the geometry of constant mean curvature one surfaces in hyperbolic space, preprint.

[UY1] M. UMEHARA AND K. YAMADA, Complete surfaces of constant mean curvature 1 in the hyperbolic 3space, Ann. of Math. 137 (1993), 611-638.

[UY2] M. UMEHARA AND K. YAMADA, A parametrization of the Weierstrass formulae and perturbation of some minimal surfaces in $\boldsymbol{R}^{3}$ into the hyperbolic 3-space, J. Reine Angew. Math. 432 (1992), 93-116.

[UY3] M. UMEHARA AND K. YAMADA, A duality on CMC-1 surfaces in the hyperbolic space and hyperbolic analogue of the Osserman inequality, Tsukuba J. Math. 21 (1997), 229-237.

[W1] Wohlgemuth, Higher genus minimal surfaces by growing handles out of a catenoid, Manuscripta Math. 70 (1991), 397-428.

[W2] Wohlgemuth, Vollstandige Minimalflachen hoheren Geschlechts und endlicher Totalkümmung, Ph. D. Thesis, University of Bonn, Bonn, 1993. 
Rua do PARQue 36, APto. 304 CEP 20940-050

RIO DE JANEIRO

BRASIL

E-mail address: costa@mat.uff.br

\section{COSTA AND V. SOUSA NETO}

Universidade Católica de Pernambuco

Departamento de Matemática

RUA do PRÍNCIPE, 526 - BoA Vista, CEP 50500-900

RECIFE-PE

BRASIL

E-mail address:vicente@unicap.br 